\title{
112. On the Mass Distribution Generated by a Function of P. L. Class
}

\author{
By Osamu ISHIKAWA \\ Mathematical Institute, Tokyo Metropolitan University \\ (Comm. by Z. SuetunA, M.J.A., July 12, 1954)
}

§1. Introduction. Let $f(x, y)$ be a subharmonic function in a planar region $G$, and $\mu(e)$ be the completely additive, non-negative Borel set function generated by $f(x, y)$. Let $c(x, y ; r)$ be the circle of radius $r$ with center $(x, y)$ included in the region $G$ with its boundary.

We shall introduce the functions:

$$
\begin{gathered}
A(f ; x, y ; r)=\frac{1}{\pi r^{2}} \int_{0}^{2 \pi} \int_{0}^{r} f(x+\rho \cos \theta, y+\rho \sin \theta) \rho d \rho d \theta, \\
I(f ; x, y ; r)=\frac{1}{2 \pi} \int_{0}^{2 \pi} f(x+r \cos \theta, y+r \sin \theta) d \theta .
\end{gathered}
$$

Saks ${ }^{11}$ proved the following important theorem:

Theorem A. If $f(x, y)$ is subharmonic in the region $G$, then, for almost all points $(x, y)$ in $G$, we have

$$
\begin{aligned}
& \lim _{r \rightarrow 0} \frac{8}{r^{2}}[A(f ; x, y ; r)-f(x, y)]=D_{s} \mu(x, y), \\
& \lim _{r \rightarrow 0} \frac{4}{r^{2}}[I(f ; x, y ; r)-f(x, y)]=D_{s} \mu(x, y),
\end{aligned}
$$

where $D_{s} \mu(x, y)$ denotes the symmetric derivative of $\mu(e)$ at $(x, y)$, that is to say,

$$
D_{s} \mu(x, y)=\lim _{\rho \rightarrow 0} \frac{\mu[C(x, y ; \rho)]}{\pi \rho^{2}},
$$

$C(x, y ; \rho)$ being the circle completely included in $G$.

Recently M. D. Reade ${ }^{2)}$ proved the following

Theorem B. If $f(x, y)$ is a function of P. L. class in G, then, for almost all points $(x, y)$ in $G$, we have

$$
\lim _{r \rightarrow 0} \frac{4}{r^{2}}\left[I^{2}(f ; x, y ; r)-A\left(f^{2} ; x, y ; r\right)\right]=f^{2}(x, y) D_{s} \sigma(x, y),
$$

where $\sigma(e)$ denotes the mass distribution generated by $\log f(x, y)$. In this paper, we shall generalize this. We shall prove in $\$ 2$ some lemmas and in $\$ 3$ our main theorem.

§. We prove some lemmas which will be used in $\S 3$. 
No. 7] On the Mass Distribution Generated by a Function of P. L. Class

Lemma 1. Let $p(x, y), q(x, y)$ and $p(x, y) q(x, y)$ be subharmonic in $G$, and put

$$
\begin{aligned}
A(p q ; x, y ; r)=\frac{1}{\pi r^{2}} \int_{0}^{2 \pi} \int_{0}^{r} p(x+\rho \cos \theta, y+\rho \sin \theta) q(x+\rho \cos \theta, \\
y+\rho \sin \theta) \rho d \rho d \theta .
\end{aligned}
$$

Further, let $\mu_{p}(e), \mu_{q}(e), \mu_{p q}(e)$ be the mass distributions generated by $p(x, y), q(x, y)$ and $p(x, y) q(x, y)$ respectively.

Then we have

$$
\begin{aligned}
\lim _{r \rightarrow 0}[L(p ; x, y ; r) L(q ; x, y ; r)-A(p q ; x, y ; r)] / r^{2} \\
\quad=\frac{q(x, y) D_{s} \mu_{p}(x, y)+p(x, y) D_{s} \mu_{q}(x, y)}{4}-\frac{1}{8} D_{s} \mu_{p q}(x, y),
\end{aligned}
$$

a.e. in $G$.

Proof. By the definitions we have

and then

$$
\begin{aligned}
L(p) L(q) & -A(p q)=\frac{1}{2}\{[L(p)+p(x, y)][L(q)-q(x, y)] \\
+ & {[L(p)-p(x, y)][L(q)+q(x, y)]\}-\{A(p q)-p q\}, }
\end{aligned}
$$

$$
\begin{aligned}
\frac{L(p) L(q)-A(p q)}{r^{2}}= & \frac{1}{8}[L(p)+p(x, y)] \frac{4}{r^{2}}[L(q)-q(x, y)] \\
& +\frac{1}{8}[L(q)+q(x, y)] \frac{4}{r^{2}}[L(p)-p(x, y)] \\
& -\frac{1}{8} \frac{8}{r^{2}}[A(p q)-p(x, y) q(x, y)] .
\end{aligned}
$$

By Theorem A, when $r \rightarrow 0$,

$$
\begin{aligned}
& \frac{4[L(q)-q(x, y)]}{r^{2}} \rightarrow D_{s} \mu_{q}(x, y), \quad \frac{4[L(p)-p(x, y)]}{r^{2}} \rightarrow D_{s} \mu_{p}(x, y) \\
& \frac{8}{r^{2}}[A(p q)-p q] \rightarrow D_{s} \mu_{p q}(x, y)
\end{aligned}
$$

a.e. in $G$.

Since $p(x, y), q(x, y)$ are subharmonic, $L(p)$ and $L(q)$ converge to $p(x, y)$ and $q(x, y)$ respectively a.e., as $r \rightarrow 0$. Therefore by (2) and (3) wet get

$$
\begin{aligned}
\lim _{r \rightarrow 0} \frac{L(p) L(q)-A(p q)}{r^{2}} & =\frac{1}{4}\left\{p(x, y) D_{s} \mu_{q}(x, y)+q(x, y) D_{s} \mu_{p}(x, y)\right\} \\
& -\frac{1}{8} D_{s} \mu_{p q}(x, y), \quad \text { a.e. in } G,
\end{aligned}
$$

which is the required.

Lemma 2. If $p(x, y), q(x, y)$ and $p(x, y) q(x, y)$ are subharmonic in $G$, and if $e$ is a Borel set completely included in $G$, then we have 


$$
\begin{aligned}
& \mu_{p q}(e)=\iint_{e} p(x, y) d \mu_{q}\left(e_{p}\right)+\iint_{e} q(x, y) d \mu_{p}\left(e_{p}\right)
\end{aligned}
$$

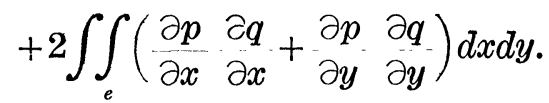

Proof. Let $D$ be an arbitrary domain such that $\bar{D} \subset G$.

Evans $^{3)}$ and Riesz ${ }^{4)}$ proved the following facts: If we put

$$
\begin{aligned}
& A_{2}(p ; x, y ; r) \equiv A(A(p) ; x, y ; r), A_{3}(p ; x, y ; r) \equiv A\left(A_{2}(p) ; x, y ; r\right), \\
& A_{2}(q ; x, y ; r) \equiv A(A(q) ; x, y ; r), A_{3}(q ; x, y ; r) \equiv A\left(A_{2}(q) ; x, y ; r\right),
\end{aligned}
$$

then there exists a positive decreasing sequence $\left\{\rho_{n}\right\}(\rho \downarrow 0)$ such that the three sequences

$$
\mu_{p}^{(n)}(e)=\iint_{e} \Delta A_{3}\left(p ; x, y ; \rho_{n}\right) d x d y, \mu_{q}^{(n)}(e)=\iint_{e} \Delta A_{3}\left(q ; x, y ; \rho_{n}\right) d x d y
$$

and

$$
\mu_{p q}^{(n)}(e)=\iint_{e} \Delta\left[A_{3}\left(p ; x, y ; \rho_{n}\right) \times A_{3}\left(q ; x, y ; \rho_{n}\right)\right] d x d y
$$

converge to $\mu_{p}(e), \mu_{q}(e), \mu_{p q}(e)$, respectively as $n \rightarrow \infty$, where $e$ denotes an open set $e(\ddot{e} \subset G)$ and is $\mu_{p^{-}}, \mu_{q^{-}}, \mu_{p q^{-}}$regular.

Now let $R$ denote orientated, $\mu_{p^{-}}, \mu_{q^{-}}, \mu_{p q^{-}}$regular rectangle contained in $D$ and put $A_{3}\left(p ; x, y ; \rho_{n}\right) \equiv \mathfrak{Y}^{(n)}(x, y), A_{3}(q ; x, y ; r) \equiv \mathfrak{B}^{(n)}(x, y)$. Let us now estimate $\Delta\left(\mathfrak{U}^{(n)} \mathfrak{B}^{(n)}\right)$. Since

$$
\begin{aligned}
& \frac{\partial^{2} \mathfrak{Y}(n) \mathfrak{B}^{(n)}}{\partial x^{2}}=\mathfrak{Y}_{x x}^{(n)}\left(\mathfrak{B}^{(n)}+2 \mathfrak{H}_{x}^{(n)} \mathfrak{B}_{x}^{(n)}+\mathfrak{H}_{x x}^{(n)} \mathfrak{B}^{(n)},\right. \\
& \frac{\partial^{2} \mathfrak{Y}(n) \mathfrak{B}^{(n)}}{\partial y^{2}}=\mathfrak{H}\left(n ; \mathfrak{B}_{y y}^{(n)}+2 \mathfrak{Y}_{y}^{(n)} \mathfrak{B}_{y}^{(n)}+\mathfrak{I}_{y y}^{(n) \mathfrak{B}^{(n)}},\right.
\end{aligned}
$$

we have

$$
\begin{aligned}
\Delta\left(\mathfrak{H}^{(n)} \mathfrak{B}^{(n)}\right) & =\mathfrak{U}^{(n)}\left(\mathfrak{B}_{x x}^{(n)}+\mathfrak{B}_{y y}^{(n)}\right)+\mathfrak{B}^{(n)}\left(\mathfrak{H}_{x x}^{(n)}+\mathfrak{P}_{y y}^{(n)}\right)+2\left(\mathfrak{H}_{x}^{(n)} \mathfrak{B}_{x}^{(n)}+\mathfrak{H}_{y}^{(n)} \mathfrak{B}_{y}^{(n)}\right) \\
& =\mathfrak{A}^{(n)} \Delta \mathfrak{B}^{(n)}+2\left(\mathfrak{H}_{x}^{(n)} \mathfrak{B}_{x}^{(n)}+\mathfrak{H}_{y}^{(n)} \mathfrak{B}_{y}^{(n)}\right)+\mathfrak{B}^{(n)} \Delta \mathfrak{U}^{(n)} .
\end{aligned}
$$

By (4), (5), (6), we obtain

$$
\begin{aligned}
\mu_{p q}(R)=\lim _{n \rightarrow \infty} \iint_{R} \mathfrak{Y}^{(n)} \Delta \mathfrak{B}^{(n)} d x d y & +\lim _{n \rightarrow \infty} \iint_{\boldsymbol{R}} \mathfrak{B}^{(n)} \Delta \mathfrak{U}^{(n)} d x d y \\
& +2 \lim _{n \rightarrow \infty} \iint_{\boldsymbol{R}}\left(\mathfrak{H}_{x}^{(n)} \mathfrak{B}_{x}^{(n)}+\mathfrak{A F}_{y}^{(n)} \mathfrak{B}_{y}^{(n)}\right) d x d y
\end{aligned}
$$

and by (4) and (5),

$$
\begin{aligned}
\mu_{p q}(R)=\lim _{n \rightarrow \infty} \iint_{R} \mathfrak{F}^{(n)} d \mu_{q}^{(n)}\left(e_{p}\right)+ & \lim _{n \rightarrow \infty} \iint_{\mathcal{R}} \mathfrak{B}^{(n)} d \mu_{p}^{(n)}\left(e_{p}\right) \\
& +2 \lim _{n \rightarrow \infty} \iint_{\boldsymbol{R}}\left(\mathfrak{H}_{x}^{(n)} \mathfrak{B}_{x}^{(n)}+\mathfrak{H}_{y}^{(n)} \mathfrak{B}_{y}^{(n)}\right) d x d y .
\end{aligned}
$$

After Frostman, ${ }^{5)}$ we have 


$$
\begin{aligned}
& \lim _{n \rightarrow \infty} \iint_{R} \mathfrak{A}^{(n)} d \mu_{q}^{(n)}\left(e_{p}\right)=\iint_{R} p(x, y) d \mu_{q}\left(e_{p}\right), \\
& \lim _{n \rightarrow \infty} \iint_{R} \mathfrak{B}^{(n)} d \mu_{p}^{(n)}\left(e_{p}\right)=\iint_{R} q(x, y) d \mu_{p}\left(e_{p}\right),
\end{aligned}
$$

and after Evans ${ }^{3)}$

$$
\begin{aligned}
& \lim _{n \rightarrow \infty} \iint_{\boldsymbol{n}}\left[\frac{\partial \mathfrak{Y}^{(n)}}{\partial x} \frac{\partial \mathfrak{B}^{(n)}}{\partial x}+\frac{\partial \mathfrak{Y}^{(n)}}{\partial y} \frac{\partial \mathfrak{B}^{(n)}}{\partial y}\right] d x d y
\end{aligned}
$$

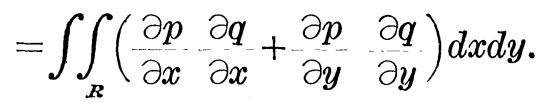

By (7), (8), (9), we get the following relation.

$$
\begin{aligned}
\mu_{p q}(R)=\iint_{\boldsymbol{R}} p(x, y) d \mu_{q}\left(e_{p}\right) & +\iint_{\boldsymbol{R}} q(x, y) d \mu_{p}\left(e_{p}\right) \\
& +2 \iint_{\boldsymbol{R}}\left(\begin{array}{cc}
\partial p & \frac{\partial q}{\partial x} \\
\partial x
\end{array}+\frac{\partial p}{\partial y} \frac{\partial q}{\partial y}\right) d x d y .
\end{aligned}
$$

By the reasoning of Reade, ${ }^{2)}$ we can see that this relation holds good for any open orientated rectangle contained in $D$.

Hence the relation (10) holds good for any Borel set contained in $D$. Since $D$ is an arbitrary domain $(\bar{D} \subset G)$, Lemma 2 is completely proved.

§3. We can show now the main theorem:

Theorem 1. If $p(x, y), q(x, y)$ and $p(x, y) q(x, y)$ are subharmonic in a domain $G$, and if $\mu_{p}(e), \mu_{q}(e)$ are mass distributions generated by $p(x, y), q(x, y)$ respectively, then we have

$$
\begin{aligned}
& \lim _{r \rightarrow 0} \frac{4}{r^{2}}[L(p ; x, y ; r) L(q ; x, y ; r)-A(p q ; x, y ; r)] \\
& =\frac{1}{2}\left[p d_{s} \mu_{q}+q d_{s} \mu_{p}\right]-\left(\begin{array}{ccc}
\partial p & \partial q \\
\partial x & \partial x
\end{array}+\frac{\partial p}{\partial y} \frac{\partial q}{\partial y}\right), \quad \text { a.e. in } G .
\end{aligned}
$$

Proof. By Lemma 1, we get

$$
\begin{aligned}
\lim _{r \rightarrow 0} \frac{4}{r^{2}}[L(p ; x, y ; r) L(q ; x, y ; r)-A(p q ; x, y ; r)] & \\
& =p D_{s} \mu_{q}+q D_{s} \mu_{q}-\frac{1}{2} D_{s} \mu_{p q}, \quad \text { a.e. in } G,
\end{aligned}
$$

and by Lemma 2

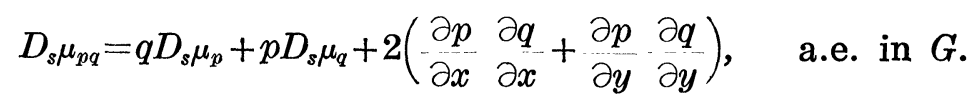

Therefore the required result is immediately obtained.

§4. We shall assume that $p(x, y), q(x, y)$ are functions of P. L. class in $G$. Then $u(x, y)=\log p(x, y)$ and 
$v(x, y)=\log q(x, y)$ are subharmonic in $G$. Let $\sigma_{p}(e)$ and $\sigma_{q}(e)$ denote the mass distributions generated by $u(x, y)$ and $v(x, y)$ respectively.

By a theorem of Beckenbach, if $p(x, y), q(x, y)$ are functions of P. L. class, then we have for any circle $C(x, y ; r)$ completely included in $G$,

$$
A(p q ; x, y ; r) \leqq L(p ; x, y ; r) L(q ; x, y ; r) .
$$

We shall discuss the value of

$$
\lim _{r \rightarrow 0} \frac{4}{r^{2}}[L(p ; x, y ; r) L(q ; x, y ; r)-A(p q ; x, y ; r)]
$$

in terms of $\sigma_{p}(e)$ and $\sigma_{q}(e)$. For this purpose we need a lemma which was proved by M. D. Reade. ${ }^{2)}$

Lemma 3. If $e$ is a Borel set $(\bar{e} \subset R)$ and $p(x, y)$ is a function of P. L. class $(\log p(x, y)=u(x, y))$, then we have

$$
\mu_{p}(e)=\iint_{e} \exp u(x, y) d \sigma\left(e_{p}\right)+\iint_{e}\left[\left(\frac{\partial u}{\partial x}\right)^{2}+\left(\frac{\partial u}{\partial y}\right)^{2}\right] p(x, y) d x d y .
$$

Therefore

$$
D_{s} \mu_{p}(x, y)=p(x, y) D_{s} \sigma_{p}(x, y)+p\left[\left(\frac{\partial u}{\partial x}\right)^{2}+\left(\frac{\partial u}{\partial y}\right)^{2}\right] .
$$

Similarly

$$
D_{s} \mu_{q}(x, y)=q(x, y) D_{s} \sigma_{q}(x, y)+q\left[\left(\frac{\partial v}{\partial x}\right)^{2}+\left(\frac{\partial v}{\partial y}\right)^{2}\right] .
$$

Hence we have the following

Theorem 2. If $p(x, y), q(x, y)$ are positive functions of P.L. class in $G$ and if $\sigma_{p}(e), \sigma_{q}(e)$ are mass distributions generated by $\log p(x, y)$ and $\log q(x, y)$, respectively, then we have

$$
\begin{aligned}
\lim _{r \rightarrow 0} & \frac{4}{r^{2}}[L(p ; x, y ; r) L(q ; x, y ; r)-A(p q ; x, y ; r)]=\frac{1}{2} p q\left\{D_{s} \sigma_{p}(x, y)\right. \\
& \left.+D_{s} \sigma_{q}(x, y)\right\}+\frac{1}{p q}\left\{\left(q \frac{\partial p}{\partial x}-p \frac{\partial q}{\partial x}\right)^{2}+\left(q \frac{\partial p}{\partial y}-p \frac{\partial q}{\partial y}\right)^{2}\right\}, \quad \text { a.e. in } G .
\end{aligned}
$$

Proof. By Theorem I, we get

$$
\begin{aligned}
P= & \lim _{r \rightarrow 0} \frac{4}{r^{2}}[L(p ; x, y ; r) L(q ; x, y ; r)-A(p q ; x, y ; r)] \\
=\frac{1}{2}\left[p(x, y) D_{s} \mu_{q}(x, y)\right. & \left.+q(x, y) D \mu_{p}(x, y)\right] \\
& \quad-\left(\frac{\partial p}{\partial x} \frac{\partial q}{\partial x}+\frac{\partial p}{\partial y} \frac{\partial q}{\partial y}\right), \quad \text { a.e. in } G .
\end{aligned}
$$

(11) and (12) give us 
No. 7] On the Mass Distribution Generated by a Function of P. L. Class

$$
\begin{aligned}
& P=\frac{1}{2}\left[p\left\{q D_{s} \sigma_{q}+q\left(\begin{array}{c}
\partial \log q \\
\partial x
\end{array}\right)^{2}+q\left(\frac{\partial \log q}{\partial y}\right)^{2}\right\}\right. \\
& \left.+q\left\{p D_{s} \sigma_{p}+p\left(\frac{\partial \log p}{\partial x}\right)^{2}+q\left(\frac{\partial \log p}{\partial y}\right)^{2}\right\}\right]-\left(\frac{\partial p}{\partial x} \frac{\partial q}{\partial x}+\frac{\partial p}{\partial y} \frac{\partial q}{\partial y}\right) \\
& =\frac{1}{2}\left[p q\left(D_{s} \sigma_{p}+D_{s} \sigma_{q}\right)+\frac{q}{p}\left\{\left(\frac{\partial p}{\partial x}\right)^{2}+\left(\frac{\partial p}{\partial y}\right)^{2}\right\}+\frac{p}{q}\left\{\left(\frac{\partial q}{\partial y}\right)^{2}+\left(\frac{\partial q}{\partial y}\right)^{2}\right\}\right] \\
& -\left(\frac{\partial p}{\partial x} \frac{\partial q}{\partial x}+\frac{\partial p}{\partial y} \frac{\partial q}{\partial y}\right) \\
& =\frac{1}{2} p q\left(D_{s} \sigma_{p}+D_{s} \sigma_{q}\right)+\frac{1}{p q}\left\{\left(q \frac{\partial p}{\partial x}-p \frac{\partial q}{\partial x}\right)^{2}+\left(q \frac{\partial p}{\partial y}-p \frac{\partial q}{\partial y}\right)^{2}\right\},
\end{aligned}
$$

which is the required.

\section{References}

1) S. Saks: On the operators of Blaschke and Privaloff for harmonic functions, Recueil Math., 9, 451-456 (1941).

2) M. D. Reade: Functions having subharmonic logarithms, Bull. American Math. Soc., 53, 89-95 (1947).

3) G. C. Evans: On potentials of positive mass, Trans. American Math. Soc., 37, 226-253 (1935).

4) F. Riesz: Sur les fonctions subharmoniques et leur rapport à la théorie du potential, Acta Math., 54, 321-360 (1930).

5) O. Frostman: Potential d'équilibre et capacité des ensembles, Lunds, Thèse (1935). 